\title{
Variación en loci isoenzimáticos entre machos y hembras de Lutzomyia shannoni (Dyar) (Diptera: Psychodidae) de Colombia
}

\author{
Estrella Cárdenas ${ }^{1}$, Leonard E. Munstermann ${ }^{2}$, Cristina Ferro ${ }^{1}$ \\ ${ }^{1}$ Laboratorio de Entomología, Instituto Nacional de Salud, Bogotá, D.C., Colombia. \\ ${ }^{2}$ Department of Epidemiology and Public Health, Yale University School of Medicine, New Haven, Conn., U.S.A.
}

Se analizaron por sexo poblaciones silvestres de Lutzomyia shannoni de tres localidades distantes entre sí: Palambí (Nariño), Cimitarra (Santander) y Chinácota (Norte de Santander), con el fin de establecer la variación en 11 isoenzimas. Estas muestras se compararon con ejemplares de una colonia mantenida en el Laboratorio de Entomología del INS desde 1992. Se utilizó el sistema de electroforesis vertical en geles de poliacrilamida al $6 \%$. Se encontró una heterocigosidad promedio entre 18,5 y $24,7 \%$ en las hembras silvestres y entre 13,5 y $19,4 \%$ entre los machos silvestres. La heterocigosidad promedio en las hembras de la colonia fue de $14,8 \%$ mientras que en los machos fue de $20,1 \%$. Se detectaron entre 2,0 y 2,5 alelos por locus. La distancia genética de Nei entre las poblaciones fue baja y osciló entre 0,005 y 0,073 . En la muestra de la colonia de 79 individuos, el locus Gpi fue homocigoto en todas las hembras y heterocigoto en todos los machos. Aunque esta observación es probablemente una consecuencia de la colonización, indica que el locus Gpi está fuertemente unido a los cromosomas que determinan el sexo, con el alelo $G p^{p, 62}$ unido al locus que determina hembras y el alelo $G p i^{p .72}$ asociado con el locus que determina machos.

Palabras clave: flebotomíneos, Lutzomyia shannoni, isoenzimas, electroforesis, variabilidad genética.

\section{Variation at isoenzyme loci among males and females of Lutzomyia shannoni (Dyar) (Diptera: Psychodidae) from Colombia}

Forest populations of Lutzomyia shannoni from 3 widely separated locations in Colombia: Palambí (Nariño), Cimitarra (Santander) and Chinácota (Norte de Santander) were analyzed for the variation at 11 isoenzymes by sex. These samples were compared with a laboratory colony maintained at the Entomology Laboratory (INS) since 1992. The vertical electrophoresis system in $6 \%$ polyacriylamide gels was used for the analysis. The mean heterozygosity among forest females ranged from 18.5 to $24.7 \%$, and among forest males from 13.5 to $19.4 \%$. The mean heterozygosity in the colony females was $14.8 \%$, whereas in males it was $20.1 \%$, and 2.0 to 2.5 alleles were detected per locus. Nei's genetic distances among the populations were low, ranging from 0.005 to 0.073 . In the colony sample of 79 individuals, the Gpi locus was homozygous in all females and heterozygous in all males. Although this is probably a consequence of colonization, it indicates that Gpi locus is tightly linked to the sex determining mechanism, with the $G p p^{p, 62}$ allele on the female locus and the $G p i^{0,72}$ allele associated with the male locus.

Key words: sand flies, Lutzomyia shannoni, isoenzyme, electrophoresis, genetic variability.

En las Américas, se han informado más de 350 especies de flebotomíneos, pero sólo se ha

Correspondencia: Cristina Ferro cferro@hemagogus.ins.gov.co

Recibido: 14/01/00; aceptado: 11/09/00 examinado cuidadosamente la estructura genética de poblaciones del vector de la leishmaniasis visceral, Lutzomyia longipalpis, entre poblaciones locales (1-3) y entre poblaciones ampliamente separadas (4-6). El flebótomo Lutzomyia shannoni, es una especie zoofílica que tiene amplia 
distribución en el hemisferio occidental. Su hábitat es principalmente el bosque y se encuentra en una región que va desde el sureste de Estados Unidos hasta el norte de Argentina. Esta especie es vector del virus de estomatitis vesicular (VSV) $(7,8)$ y, en condiciones de laboratorio, puede soportar el desarrollo de tres especies de Leishmania, por lo menos: L. mexicana (9), L. chagasi (10) y L. panamensis (Ferro et al., datos no publicados).

En Colombia, L. shannoni tiene una amplia distribución. Se ha registrado en los siguientes departamentos: Antioquia, Bolivar, Boyacá, Caldas, Caquetá, Chocó, Cundinamarca, La Guajira, Magdalena, Norte de Santander, Santander, Tolima y Valle $(11,12)$. Por su parte, en 1996, el Instituto Colombiano Agropecuario (ICA) registró 680 bovinos infectados con el serotipo New Jersey de VSV y más de 300 infectados con el serotipo Indiana en más de 100 municipios (13). Coincidencialmente, los municipios registrados por el ICA con estomatitis vesicular (EV) se encuentran ubicados en los departamentos donde se ha registrado $L$. shannoni $(11,12)$. Esto sugiere la posibilidad de que esta especie esté involucrada en el ciclo enzoótico del virus de EV en nuestro país. Además, Tesh y colaboradores (14) encontraron el serotipo Alagoas del virus de EV en L. shannoni y otras especies de Lutzomyia.

Estos hallazgos motivaron nuestro interés por realizar estudios básicos de biología y genética de L. shannoni en Colombia (15-18), puesto que el estudio de la variabilidad genética y la biología reproductiva de artrópodos vectores es fundamental para el entendimiento del papel que desempeñan en la epidemiología de las enfermedades. Entre los factores biológicos que afectan la tasa de transmisión de enfermedades transmitidas por artrópodos, se encuentran los siguientes: tasa de fecundidad, tasa de mortalidad, densidad, distribución por edades, tasa de migración y variación genética $(19,20)$.

Cárdenas y colaboradores (18) analizaron loci isoenzimáticos en tres poblaciones silvestres y una colonia de laboratorio de L. Shannoni y estimaron la tasa de migración, discutieron su importancia epidemiológica, analizaron la frecuencias genotípicas para equilibrio de Hardy-
Weinberg y compararon la variabilidad genética entre poblaciones sin separarlas por sexos. Observaron dos genotipos en el locus Gpien los individuos de la colonia. Los machos eran heterocigotos y las hembras homocigotas. Esta observación fue básica para formularnos dos preguntas: ¿existe diferencia genética entre machos y hembras en poblaciones de $L$. shannoni? y ¿qué tan amplia es la distancia genética entre sexos de cada población?

El presente artículo describe la variación de 13 loci isoenzimáticos entre sexos de las poblaciones analizadas por Cárdenas y colaboradores (18) y se exhiben las distancias genéticas entre machos y hembras de cada población.

\section{Materiales y métodos}

\section{Sitios de muestreo}

Método de recolección y preservación de especímenes. Los flebótomos fueron recolectados por aspiración directa sobre troncos de árboles grandes entre las 10 y las 14 horas. Se recolectaron ejemplares de Lutzomyia en los siguientes sitios y fechas:

1. Plantación tradicional de café, altura $1.235 \mathrm{~m}$, Finca Experimental Blonay, Chinácota (Norte de Santander) $\left(7^{\circ} 34^{\prime} \mathrm{N} ; 72^{\circ} 37^{\prime} \mathrm{O}\right), 14$ de mayo de 1998.

2. Bosque primario, altura $68 \mathrm{~m}$, en Palambí cerca de Tumaco (Nariño) $\left(1^{\circ} 45^{\prime} \mathrm{N}, 78^{\circ} 28^{\prime} \mathrm{O}\right), 3$ de julio de 1998.

3. Bosque secundario en la hacienda San Miguel, altura $113 \mathrm{~m},\left(6^{\circ} 23^{\prime} \mathrm{N}, 74^{\circ} 21^{\prime} \mathrm{O}\right)$ cerca a Cimitarra (Santander), 26 de diciembre de 1998.

Las distancias geográficas aproximadas entre las anteriores localidades son las siguientes: Chinácota-Cimitarra, $250 \mathrm{~km}$; Chinácota-Palambí, 950 km, y Cimitarra-Palambí, 680 km. Después de la recolección, los flebótomos se inmobilizaron en nitrógeno líquido y se clasificaron preliminarmente por observaciones morfológicas externas. Luego, se separaron por sexos y se colocaron en viales de criopreservación en grupos de 50 insectos por cada vial. Inmediatamente después de la separación de los insectos, los viales se preservaron en nitrógeno líquido. 


\section{Determinación taxonómica}

Los ejemplares de campo fueron clasificados hasta especie con base en las características morfológicas de la terminalia del macho y la espermateca de la hembra, utilizando los esquemas y claves de Young y Duncan (21), para lo cual se cortaron los últimos segmentos del abdomen. El resto del cuerpo de L. shannoni se preservó individualmente en viales de microcentrífuga a $-80{ }^{\circ} \mathrm{C}$ hasta el momento de utilizarlos en el análisis de isoenzimas. Las otras especies fueron preservadas para la colección del Laboratorio.

\section{Método electroforético}

Las muestras fueron analizadas para variación en 11 isoenzimas (cuadro 1). Los adultos de $L$. shannoni se prepararon para electroforesis por homogenización individual en $10 \circ 20 \mu \mathrm{l}$ de solución tampón de macerado (23) (sucrosa al $10 \%$, Triton $\mathrm{X}-100$ al $0,1 \%$, Tris-citrato $0,02 \mathrm{M}$ y azul de bromofenol $0,002 \%$ ). Se utilizó un sistema de electroforesis vertical en geles de poliacrilamida al $6 \%$, en condiciones no desnaturalizantes.
Según la enzima por separarse, se colocó en cada bolsillo del gel entre 1 y $3 \mu \mathrm{l}$ del insecto triturado, siguiendo un procedimiento estándar (23). Se emplearon dos sistemas de soluciones tampón (tris-citrato y tris-borato-EDTA) para maximizar la separación de isoenzimas. Se utilizó un procedimiento de teñido específico para cada enzima con el fin de visualizar los fenotipos isoenzimáticos (bandas) sobre los geles (24). Las muestras puestas sobre los geles migraron del cátodo al ánodo. Al locus más lento se le designó como número 1 y al siguiente como 2 . En cada locus, las alozimas se distinguieron en orden alfabético de acuerdo al valor estimado de su movilidad relativa $(R f)(24)$. Los valores de $R f$ se calcularon utilizando la razón entre el valor de la distancia recorrida por una banda desde el origen del gel y la distancia recorrida por una banda de la enzima comercial respectiva.

\section{Análisis de datos}

Se utilizó el programa de computadora BIOSYS1 (25) para obtener las frecuencias alélicas y los valores de la variabilidad genética en las subpoblaciones respectivas.

Cuadro 1. Enzimas analizadas para variabilidad genética en poblaciones de L. shannoni.

\begin{tabular}{|c|c|c|c|c|}
\hline Enzimas & Número E.C.* & Abreviatura & $\begin{array}{l}\text { Referencia comercial } \\
(\text { Sigma })^{* *}\end{array}$ & Buffer $r^{* * *}$ \\
\hline \multicolumn{5}{|l|}{ Oxidorreductasas } \\
\hline Glicerol 3-fosfatodeshidrogenasa & 1.1.1.8 & Gpd & G-6751 & TC \\
\hline Malatodeshidrogenasa & 1.1.1.37 & Mdh & M-9004 & $\mathrm{TC}$ \\
\hline Enzima málica & 1.1.1.40 & $\mathrm{Me}$ & $M-5257$ & TBE \\
\hline Isocitratodeshidrogenasa & 1.1.1.42 & Idh & $\mathrm{I}-2002$ & $\mathrm{TC}$ \\
\hline \multicolumn{5}{|l|}{ Transferasas } \\
\hline Aspartatoaminotrasferasa & 2.6.1.1 & Aat & G-7005 & $\mathrm{TC}$ \\
\hline Hexocinasa & 2.7.1.1 & $\mathrm{Hk}$ & $\mathrm{H}-5000$ & TBE \\
\hline Argininacinasa & 2.7 .3 .3 & Ark & A-3389 & TC \\
\hline Adenylatacinasa & 2.7.4.3 & $\mathrm{Ak}$ & $M-3003$ & TC \\
\hline \multicolumn{5}{|l|}{ Hidrolasas } \\
\hline Esterasa & 3.1.1.1 & Est & $E-2884$ & TBE \\
\hline \multicolumn{5}{|l|}{ Liasas } \\
\hline Fumaratohidratasa & 4.2 .1 .2 & Fum & $\mathrm{F}-1757$ & TBE \\
\hline \multicolumn{5}{|l|}{ Isomerasas } \\
\hline Glucosa-6-fosfatoisomerasa & 5.3 .1 .9 & Gpi & P-5381 & TBE \\
\hline
\end{tabular}

* Número de clasificación de enzimas propuesto por el Enzyme Commission Classification System (22).

** Referencias comerciales utilizadas para calcular las distancias de migración relativas de las isoenzimas (Rf).

***Solución reguladora utiiizada para la separación de alozimas: TC, tris-citrato TBE, tris-borato-EDTA. 


\section{Resultados}

\section{Variabilidad genética}

Se analizaron 11 enzimas (cuadro 1). Las enzimas malato-deshidrogenasa y esterasa mostraron dos loci, para un total de 13 loci examinados en cuatro poblaciones de L. shannoni. En todas las poblaciones, 11 de los 13 loci fueron polimórficos (cuadro 2). El número de alelos por locus osciló entre 2 y 2,5 en todas las poblaciones (cuadro 3 ); no obstante, se identificaron 7 alelos en Est-2 y 4 alelos en Fum y Gpi. La muestra examinada de la colonia de laboratorio mostró 7 loci monomórficos tanto en machos como en hembras, mientras que las muestras silvestres presentaron el siguiente número de loci monomórficos: 5 en machos y 4 en hembras de Palambí, 5 en machos y 6 en hembras de Chinácota y 3 en ambos sexos de Cimitarra (cuadro 2). El locus Gpi fue homocigoto en todas las hembras de la colonia, mientras que todos los machos fueron heterocigotos (figura 1).

El porcentaje de loci polimórficos osciló entre 38,5 y $69,2 \%$. Los individuos de la colonia mostraron el menor valor, mientras que las hembras de Palambí exhibieron el mayor valor. La heterocigosidad promedio observada tuvo un valor alto en la muestra examinada de Palambí, seguida por la muestra de Chinácota. Mientras que los machos de Cimitarra y las hembras de la colonia mostraron un valor bajo para este parámetro (cuadro 3).

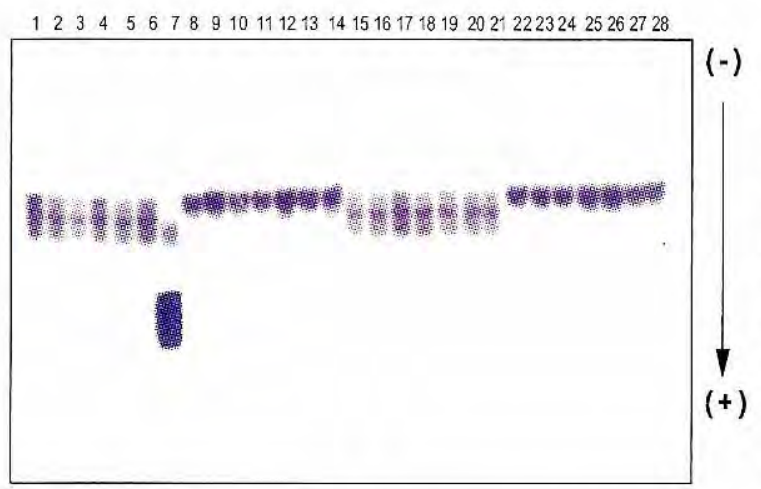

Figura 1. Zimograma de la glucosafosfatoisomerasa obtenido con ejemplares de Lutzomyia shannoni de la colonia de laboratorio. Columnas: 1-6 machos; 7: Gpi comercial; 8-14 hembras; 15-21 machos, y 22-28 hembras.

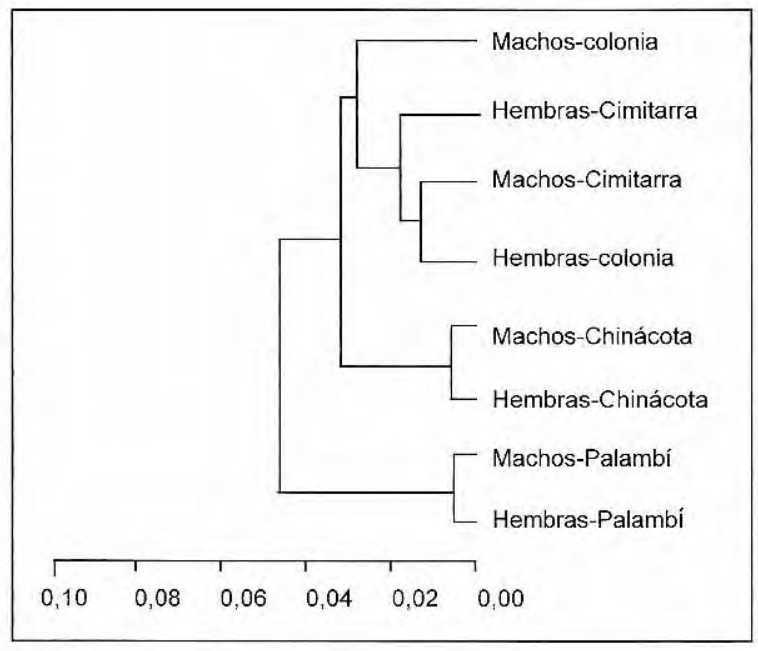

Figura 2. Dendrograma construido con el método UPGMA. Relaciones basadas en distancia genética (26) de machos y hembras de tres poblaciones silvestres y una colonia de laboratorio de Lutzomyia shannoni.

\section{Similaridad y distancia genética}

Las poblaciones de $L$. shannoni analizadas presentaron en promedio una similitud del $96,7 \%$ en los 13 loci analizados. La distancia genética (26) entre machos y hembras de Palambí y Chinácota fue de sólo 0,005 y 0,007 (cuadro 4 y figura 2). Los machos y hembras de Cimitarra y de la colonia formaron un grupo. Este grupo se separó del clado de Chinácota por una distancia genética de 0,030 , mientras que el clado de Palambí se separó de los dos grupos anteriores por una distancia genética de 0,047 (figura 2).

\section{Discusión}

Son ampliamente conocidos los estudios en genética de poblaciones de campo y laboratorio de varias especies del género Drosophila y mucho de lo conocido a nivel molecular sobre mutaciones cromosómicas, genes ligados al sexo, selección, evolución y otros aspectos, ha sido dilucidado principalmente a partir de estudios de laboratorio con Drosophila melanogaster. Aunque se han realizado varios estudios de genética basados en la variación de loci isoenzimáticos en poblaciones de L. longipalpis en Colombia $(2,5)$, en general, se encuentran pocos en flebotomíneos. Cárdenas y colaboradores (18) realizaron un primer trabajo 
Cuadro 2. Frecuencias alélicas para todos los loci en cuatro poblaciones de Lutzomyia shannoni.

\begin{tabular}{|c|c|c|c|c|c|c|c|c|c|}
\hline \multirow[b]{2}{*}{ Loci } & \multicolumn{9}{|c|}{ Población } \\
\hline & $R f$ & $\begin{array}{l}\text { Macho } \\
\text { Colonia }\end{array}$ & $\begin{array}{l}\text { Hembra } \\
\text { Colonia }\end{array}$ & $\begin{array}{l}\text { Macho } \\
\text { Palambi }\end{array}$ & $\begin{array}{l}\text { Hembra } \\
\text { Palambí }\end{array}$ & $\begin{array}{l}\text { Macho } \\
\text { Chinácota }\end{array}$ & $\begin{array}{c}\text { Hembra } \\
\text { Chinácota }\end{array}$ & $\begin{array}{l}\text { Macho } \\
\text { Cimitarra }\end{array}$ & $\begin{array}{r}\text { Hembra } \\
\text { Cimitarra }\end{array}$ \\
\hline \multicolumn{10}{|l|}{ Aat } \\
\hline $\mathrm{n}$ & & 26 & 28 & 29 & 32 & 28 & 32 & 25 & 23 \\
\hline & 009 & 1,000 & 1,000 & 0,879 & 0,828 & 0,875 & 0,875 & 0,940 & 0,913 \\
\hline & 0,15 & -0 & $-0-$ & 0,121 & 0,172 & 0,125 & 0,125 & 0,060 & 0,087 \\
\hline \multicolumn{10}{|l|}{$A k$} \\
\hline $\mathrm{n}$ & & 26 & 27 & 21 & 26 & 26 & 32 & 37 & 8 \\
\hline & 5,45 & -0 & $-0-$ & -0 & 0,096 & -0 & $-0-$ & 0,027 & -0 \\
\hline & 6,75 & 0,981 & 0,944 & 1,000 & 0,904 & 1,000 & 1,000 & 0,892 & 1,000 \\
\hline & 7,65 & 0,019 & 0,056 & $-0-$ & $-0-$ & -0 & $-0-$ & 0,081 & $-0-$ \\
\hline \multicolumn{10}{|l|}{ Ark } \\
\hline $\mathrm{n}$ & & 26 & 27 & 21 & 26 & 26 & 32 & 37 & 8 \\
\hline & 0,94 & 1,000 & 1,000 & 1,000 & 1,000 & 1,000 & 1,000 & 1,000 & 1,000 \\
\hline Est-1 & & & & & & & & . & \\
\hline $\mathrm{n}$ & & 32 & 35 & 52 & 28 & 40 & 37 & 45 & 27 \\
\hline & 0,50 & 1,000 & 1,000 & 1,000 & 1,000 & 1,000 & 1,000 & 1,000 & 1,000 \\
\hline \multicolumn{10}{|l|}{ Est-2 } \\
\hline $\mathrm{n}$ & & 28 & 35 & 47 & 30 & 41 & 38 & 41 & 26 \\
\hline & 1,50 & 0,143 & 0,186 & 0,085 & 0,017 & 0,085 & 0,079 & 0,061 & 0,038 \\
\hline & 1,58 & 0,339 & 0,229 & 0,160 & 0,167 & 0,122 & 0,066 & 0,037 & $-0-$ \\
\hline & 1,66 & 0,107 & 0,214 & 0,128 & 0,200 & 0,195 & 0,184 & 0,232 & 0,154 \\
\hline & 1,74 & 0,179 & 0,057 & 0,202 & 0,117 & 0,341 & 0,171 & 0,159 & 0,250 \\
\hline & 1,83 & 0,196 & 0,114 & 0,191 & 0,200 & 0,122 & 0,263 & 0,146 & 0,308 \\
\hline & 1,91 & 0,018 & 0,143 & 0,160 & 0,250 & 0,122 & 0,1322 & 0,293 & 0,192 \\
\hline & 2,00 & 1,018 & 0,057 & 0,074 & 0,050 & 0,012 & 0,105 & 0,073 & 0,058 \\
\hline \multicolumn{10}{|l|}{ Fum } \\
\hline n & & 27 & 29 & 14 & 20 & 17 & 26 & 34 & 29 \\
\hline & 1,20 & 0,111 & 0,241 & $-0-$ & 0,100 & 0,118 & 0,096 & 0,191 & 0,207 \\
\hline & 1,30 & 0,259 & 0,241 & 0,250 & 0,300 & 0,324 & 0,538 & 0,324 & 0,517 \\
\hline & 1,40 & 0,296 & 0,328 & 0,357 & 0,425 & 0,324 & 0,327 & 0,279 & 0,190 \\
\hline & 1,50 & 0,333 & 0,190 & 0,393 & 0,175 & 0,235 & 0,038 & 0,206 & 0,086 \\
\hline \multicolumn{10}{|l|}{ Gpd } \\
\hline $\mathrm{n}$ & & 27 & 36 & 41 & 14 & 29 & 32 & 25 & 18 \\
\hline & 1,08 & 0,722 & 0,778 & 0,683 & 0,393 & 0,466 & 0,359 & 0,960 & 0,639 \\
\hline & 1,12 & 0,278 & 0,222 & 0,244 & 0,393 & 0,328 & 0,438 & 0,040 & 0,361 \\
\hline & 1,16 & $-0-$ & $-0-$ & 0,073 & 0,214 & 0,207 & 0,203 & $-0-$ & $-0-$ \\
\hline \multicolumn{10}{|l|}{ Gpi } \\
\hline$n$ & & 39 & 40 & 25 & 34 & 32 & 36 & 48 & 26 \\
\hline & 0,50 & $-0-$ & -0 & -0 & 0,015 & $-0-$ & -0 & 0,094 & 0,135 \\
\hline & 0,62 & 0,500 & 1,000 & 0,240 & 0,294 & 0,906 & 0,972 & 0,750 & 0,731 \\
\hline & 0,72 & 0,500 & $-0-$ & 0,360 & 0,265 & 0,094 & 0,028 & 0,135 & 0,077 \\
\hline & 0,85 & $-0-$ & $-0-$ & 0,400 & 0,426 & $-0-$ & -0 & 0,021 . & 0,058 \\
\hline \multicolumn{10}{|l|}{$H k$} \\
\hline$n$ & & 26 & 28 & 21 & 27 & 26 & 29 & 44 & 20 \\
\hline & 0,77 & 1,000 & 1,000 & 0,952 & 0,907 & 1,000 & 1,000 & 0,989 & 1,000 \\
\hline & 0,83 & -0 & $-0-$ & 0,048 & 0,093 & $-0-$ & -0 & 0,011 & $-0-$ \\
\hline \multicolumn{10}{|l|}{ ldh } \\
\hline $\mathrm{n}$ & & 26 & 28 & 18 & 28 & 26 & 31 & 24 & 16 \\
\hline & 2,00 & 1,000 & 1,000 & 1,000 & 1,000 & 0,962 & 1,000 & 1,000 & 1,000 \\
\hline & 2,30 & $-0-$ & $-0-$ & $-0-$ & $-0-$ & 0,038 & $-0-$ & $-0-$ & $-0-$ \\
\hline \multicolumn{10}{|c|}{$M d h-1$} \\
\hline $\mathrm{n}$ & & 26 & 28 & 16 & 27 & 26 & 31 & 24 & 18 \\
\hline & 0,50 & 1,000 & 1,000 & 1,000 & 1,000 & 0,942 & 0,887 & 0,979 & 1,000 \\
\hline & 1,25 & -0 & $-0-$ & -0 & $-0-$ & 0,058 & 0,113 & 0,021 & $-0-$ \\
\hline
\end{tabular}


Cuadro 2 (continuación)

\begin{tabular}{|c|c|c|c|c|c|c|c|c|c|}
\hline \multirow[b]{2}{*}{ Loci } & \multirow[b]{2}{*}{$R f$} & \multicolumn{8}{|c|}{ Población } \\
\hline & & $\begin{array}{l}\text { Macho } \\
\text { Colonia }\end{array}$ & $\begin{array}{l}\text { Hembra } \\
\text { Colonia }\end{array}$ & $\begin{array}{l}\text { Macho } \\
\text { Palambí }\end{array}$ & $\begin{array}{l}\text { Hembra } \\
\text { Palambí }\end{array}$ & $\begin{array}{c}\text { Macho } \\
\text { Chinácota }\end{array}$ & $\begin{array}{l}\text { Hembra } \\
\text { Chinácota }\end{array}$ & $\begin{array}{c}\text { Macho } \\
\text { Cimitarra }\end{array}$ & $\begin{array}{l}\text { Hembra } \\
\text { Cimitarra }\end{array}$ \\
\hline \multicolumn{10}{|c|}{ Mdh-2 } \\
\hline$n$ & & 26 & 28 & 18 & 27 & 26 & 31 & 24 & 18 \\
\hline & 5,25 & 1,000 & 1,000 & 0,944 & 0,889 & 1,000 & 1,000 & 0,938 & 0,861 \\
\hline & 6,25 & $-0-$ & -0 & 0,056 & 0,111 & -0 & -0 & 0,063 & 0,139 \\
\hline \multicolumn{10}{|l|}{$M e$} \\
\hline$n$ & & 27 & 28 & 18 & 27 & 26 & 31 & 25 & 18 \\
\hline & 1,71 & 0,056 & 0,071 & 0,333 & 0,389 & 0,096 & 0,048 & $-0-$ & 0,139 \\
\hline & 1,93 & 0,704 & 0,714 & 0,556 & 0,556 & 0,596 & 0,452 & 0,960 & 0,667 \\
\hline & 2,10 & 0,241 & 0,214 & 0,111 & 0,056 & 0,308 & 0,500 & 0,040 & 0,194 \\
\hline
\end{tabular}

Rf: razón de la distancia de migración desde el origen del gel de una banda y la distancia de migración de la misma enzima comercial.

Cuadro 3. Variabilidad en 13 loci isoenzimáticos examinados en poblaciones de Lutzomyia shannoni.

\begin{tabular}{lccccc}
\hline Población & $\begin{array}{c}\text { Promedio de } \\
\text { la muestra } \\
\text { por locus }\end{array}$ & $\begin{array}{c}\text { Promedio } \\
\text { de alelos } \\
\text { por locus }\end{array}$ & $\begin{array}{c}\text { Porcentaje de loci } \\
\text { polimórficos }\end{array}$ & \begin{tabular}{c} 
Heterocigosidad promedio \\
\cline { 5 - 6 } Observada
\end{tabular} & $\begin{array}{c}\text { Esperada } \\
\text { Equi. Hardy- } \\
\text { Weinberg }\end{array}$ \\
\hline Machos-colonia & 27,8 & 2,1 & 38,5 & 20,1 & 22,6 \\
Hembras-colonia & 30,5 & 2,0 & 38,5 & 14,8 & 19,2 \\
Machos-Palambi & 26,2 & 2,3 & 53,8 & 19,4 & 28,3 \\
Hembras-Palambí & 26,6 & 2,5 & 69,2 & 24,7 & 32,8 \\
Machos-Chinácota & 28,4 & 2,3 & 53,8 & 17,2 & 25,6 \\
Hembras-Chinácota & 32,2 & 2,2 & 46,2 & 18,5 & 24,0 \\
Machos-Cimitarra & 33,3 & 2,5 & 46,2 & 13,5 & 20,3 \\
Hembras-Cimitarra & 19,6 & 2,2 & 53,8 & 18,8 & 25,3 \\
\hline
\end{tabular}

* Un locus se considera polimórfico cuando la frecuencia del alelo más común no excede de 0,95.

sobre genética de poblaciones de L. shannonie informaron sobre la unión de los alelos $G p^{i, 62}$ y $\mathrm{Gpi}^{0,72}$ a los cromosomas que determinan el sexo en los individuos de la colonia. Esta característica no se observó en las poblaciones silvestres. Es muy probable que durante la colonización de $L$. shannoni el alelo $G p i^{0,72}$ fuera atrapado por el locus que determina machos y el alelo $G p^{0,62}$ por el locus que determina hembras (figura 1). Esto explica la heterocigosidad total para este locus en los machos y la homocigosidad total en las hembras de la colonia. Como consecuencia de esta fuerte unión, el locus Gpi forzó la heterocigosidad en los machos de la colonia e incrementó artificial-mente su heterocigosidad promedio (cuadro 3). La unión de alelos a los cromosomas sexuales por proceso de colonización y endocría no es común en otros dípteros (27).
Para dar respuesta a las preguntas formuladas en la introducción, tenemos lo siguiente: al analizar por separado machos y hembras en las cuatro poblaciones (cuadro 4, figura 2), se encontró que no existe diferencia entre machos y hembras de las poblaciones de Palambí y Chinácota, de acuerdo con lo esperado entre sexos de la misma especie. La pequeña distancia genética observada $(0,005$ y 0,007$)$ se debe únicamente a la separación de machos y hembras como poblaciones diferentes (efecto Wahlund) (20). Esto indica que machos $y$ hembras en estas dos poblaciones son genéticamente similares.

En cuanto a las poblaciones de Cimitarra y la colonia se tiene lo siguiente: los machos de Cimitarra se agruparon con las hembras de la colonia y mostraron una distancia genética de 0,014 . Las hembras de Cimitarra se separaron del 
Cuadro 4. Indices distancia genética Nei (26) en poblaciones de Lutzomyia shannoni.

\begin{tabular}{|c|c|c|c|c|c|c|c|c|}
\hline Población & 1 & 2 & 3 & 4 & 5 & 6 & 7 & 8 \\
\hline 1. Macho-colonia & ----- & 0,025 & 0,018 & 0,038 & 0,026 & 0,052 & 0,029 & 0,027 \\
\hline 2. Hembra-colonia & & - & 0,053 & 0,060 & 0,012 & 0,029 & 0,014 & 0,017 \\
\hline 3. Macho-Palambí & & & 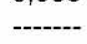 & 0,005 & 0,043 & 0,073 & 0,046 & 0,037 \\
\hline 4. Hembra-Palambí & & & & - & 0,041 & 0,059 & 0,061 & 0,035 \\
\hline 5. Macho-Chinácota & & & & & ---- & 0,007 & 0,032 & 0,011 \\
\hline 6. Hembra-Chinácota & & & & & & -n- & 0,061 & 0,018 \\
\hline 7. Macho-Cimitarra & & & & & & & ---- & 0,018 \\
\hline 8. Hembra-Cimitarra & & & & & & & & - \\
\hline
\end{tabular}

clado machos-Cimitarra y hembras-colonia por una distancia genética de 0,017, y los machos de la colonia se separaron del grupo anterior por una distancia genética de 0,027 . La distancia genética entre sexos de los individuos analizados de la colonia fue de 0,025 y entre sexos de los individuos analizados de Cimitarra fue de 0,018 . Esto sugiere que, en estas dos poblaciones, hay tendencia a presentar distanciamiento genético entre sexos. El agrupamiento entre machos-Cimitarra con hembras-colonia mostró una estrecha relación entre estas dos poblaciones. Esta observación permite pensar que la colonia pudo haberse originado de fundadores que provenían del bosque Los Corales cercano al bosque de San Miguel, como anotaron Cárdenas y colaboradores(18).

En conclusión, la variación alozímica demostró, en primer lugar, que no existe variación genética entre sexos de algunas poblaciones silvestres de L. shannoni como lo observado en las poblaciones de Chinácota y Palambí. En segundo lugar, se demostró que, entre machos y hembras de la colonia, existe dimorfismo sexual marcado por el locus Gpi, y, por último, se demostró que los individuos de la colonia están muy relacionados con la población de Cimitarra.

Como se puede apreciar, estos resultados amplían el conocimiento en genética de poblaciones de otras especies de insectos diferentes a las del género Drosophila.

\section{Agradecimientos}

Al Instituto Nacional de Salud de Colombia, proyecto No. 19973100302 del Laboratorio de Entomología y a The United States National Institutes of Health (AI-34521 to LEM), por la financiación de este trabajo. Al señor Marco Fidel
Suárez, auxiliar del Laboratorio de Entomología INS, por la colaboración en la recolección del material entomológico.

\section{Referencias}

1. Morrison AC, Ferro C, Pardo R, Torres $\mathbf{M}$, Munstermann LE. An ecological and genetic study of Lutzomyia longipalpis in a central Colombian focus of visceral leishmaniasis. Bol Dir Malariol, Saniamiento Ambiental 1995;35:235-48.

2. Munstermann LE, Ferro $C$, Pardo $R$, Torres $M$, Morrison AM. Genetic structure of local populations of Lutzomyia longipalpis (Diptera: Psychodidae) in Central Colombia. J Med Entomol 1998;35:82-9.

3. Mutebi JP, Rowton E, Herrero MV, Ponce C, Belli A, Valle S, et al. Genetic variability among populations of the sand fly Lutzomyia (Lutzomyia) longipalpis (Diptera: Psychodidae) from Central America. J Med Entomol 1998;35:169-74.

4. Mukhopadhyay J, Ghosh K, Rangel E, Munstermann LE. Genetic variability in biochemical characters of Brazilian field populations of the Leishmania vector, Lutzomyia longipalpis (Diptera: Psychodidae). Am J Trop Med Hyg 1998;59:893-901.

5. Lanzaro GC, Alexander B, Mutebi JP, Montoya J, Warburg A. Genetic variation among natural and laboratory colony populations of Lutzomyia longipalpis (Lutz \& Neiva, 1912) (Diptera: Psychodidae) from Colombia. Mem Inst Oswaldo Cruz 1998;93:65-9.

6. Mutebi JP, Alexander B, Sherlock I, Wellington J, Souza AA, Shaw J, et al. Breeding structure of the sand fly Lutzomyia longipalpis (Lutz \& Neiva) in Brazil. Am J Trop Med Hyg 1999;61:149-57.

7. Comer JA, Tesh RB, Modi GB, Corn JL, Nettles VF. Vesicular stomatitis virus, New Jersey serotype: Replication in and transmission by Lutzomyia shannoni (Diptera: Psychodidae). Am J Trop Med Hyg 1990;42: 483-90.

8. Comer JA, Stallknecht DE, Corn JL, Nettles VF. Lutzomyia shannoni (Diptera: Psychodidae): a biological vector of the New Jersey serotipe of Vesicular Stomatitis Virus on Ossabaw Island, Georgia. Parassitologia 1991; 33:151-8. 
9. Lawyer PG, Young DG, Butler JF, Akin DE. Development of Leishmania mexicana in Lutzomyia diabolica and Lutzomyia shannoni (Diptera: Psychodidae). J Med Entomol 1987;24:347-55.

10. Endris RG, Perkins PV, Young DG, Johnson RN. Techniques for laboratory rearing of sand flies (Diptera: Psychodidae). Mosquito News 1982;42:400-7.

11. Young DG. A review of the bloodsucking Psychodid Flies of Colombia (Diptera: Phlebotominae and Sycoracinae). Agricult. Experi. Stat. Institute of Food and Agricultural Sciences. Gainesville: University of Florida; 1979. p.1-266.

12. Montoya-Lerma J, Ferro C. Flebótomos (Diptera: Psychodidae) de Colombia. En: Insectos de Colombia. Santa Fe de Bogotá: Academia Colombiana de Ciencias Exactas, Físicas y Naturales. Colección Jorge Alvarez Lleras; 1999. p.2:211-45.

13. Instituto Colombiano Agropecuario. Boletín de sanidad animal 1996-1997. Grupo de Epidemiología Veterinaria, División de Sanidad Animal, Instituto Colombiano Agropecuario. Bogotá, Colombia; 1999 (en prensa).

14. Tesh RB, Boshell J, Modi GB, Morales A, Young DG, Corredor A, et al. Natural infection of humans, animals and phlebotomine sand flies with the Alagoas serotype of vesicular estomatitis virus in Colombia. Am J Trop Med Hyg 1987;36:653-61.

15. Ferro C, Cárdenas E, Corredor D, Morales A, Munstermann LE. Life cycle and fecundity analysis of Lutzomiya shannoni (Dyar) (Diptera: Psychodidae). Mem Inst Oswaldo Cruz 1998;93:195-9.

16. Cárdenas E. Biología reproductiva bajo condiciones experimentales y variabilidad genética de Lutzomyia shannoni (Dyar) (Diptera: Psychodidae) (tesis). Santa Fe de Bogotá: Universidad Nacional de Colombia; 1999.

17. Cárdenas E, Ferro C, Corredor D, Martínez O, Munstermann LE. Reproductive biology of Lutzomyia shanonni (Dyar) (Diptera: Psychodidae) under experimental conditions. J Vect Ecol 1999;24:158-70.
18. Cárdenas E, Munstermann LE, Martínez $\mathrm{O}$, Corredor D, Ferro C. Genetic variability among populations of Lutzomyia shannoni (Dyar) (Diptera: Psychodidae) from Colombia. Mem Inst Oswaldo Cruz 2001 (en prensa).

19. Black IV WC, Moore CH G. Population biology as a tool for studying vector-borne diseases. In: Beaty BJ, Marquardt WC, editors. The biology of disease vectors. Boulder, CO: University Press of Colorado; 1996. p.1-632.

20. Tabachnick WJ, Black IV WC. Population genetics in vector biology. In: The biology of disease vectors. Beaty BJ, Marquardt WC, editors. Boulder, CO: University Press of Colorado; 1996. p.1-632.

21. Young DG, Duncan MA. Guide to the identification and geographic distribution of Lutzomyia sand flies in Mexico, the West Indies, Central and South America (Diptera: Psychodidae). Gainesville, Florida: American Entomology Institute; 1994. p.1-881.

22. Barman TE. Enzime handbook. Vol. 1 and Vol. 2. Second printing. New York, Berlin, Heidelberg, Tokio: SpringerVerlag; 1985. p.1-928

23. Munstermann LE. Electrophoresis procedures. Sand Fly Genetics Program. New Haven, CT: Yale University School of Medicine; 1994. p.1-31.

24. May B. Starch gel electrophoresis of allozimes. In: Molecular genetic analysis of population. A practical approach. Oxford: IRL, Oxford University Press; 1992. p. $1-280$.

25. Swofford DL, Selander RB. BIOSYS-1: a FORTRAN program for the comprehensive analysis of electrophoretic data in population genetics and systematics. J Hered 1981;72:281-3.

26. Nei M. Estimation of average heterozygosity and genetic distance from a small number of individuals. Genetics 1978;89:583-90.

27. Munstermann LE. Unexpected genetic consequences of colonization and inbreeding: allozyme tracking in Culicidae (Diptera). Ann Entomol Soc Am 1994;87:157-64. 